\title{
La pisciculture des Pangasiidae
}

Jérôme Lazard ${ }^{1}$

Philippe Cacot ${ }^{1}$

Jacques Slembrouck ${ }^{2}$

Marc Legendre ${ }^{2}$

1 Cirad

UR «Aquaculture et gestion des ressources

aquatiques"

TA B-20/01

Avenue Agropolis

34398 Montpellier cedex 5

France

$<$ jerome.lazard@cirad.fr>

<phcacot@gmail.com>

2 IRD UR CAVIAR

Gamet-IRD/Cemagref

BP 5095

361 rue Jean-François Breton

34196 Montpellier cedex 5

France

<jacques.slembrouck@ird.fr> $<$ marc.legendre@ird.fr>

\section{Résumé}

L'évolution de la production de Pangasiidae depuis 15 ans en Asie du Sud-Est est celle d'une filière en pleine expansion. Le Vietnam offre l'exemple abouti d'une dynamique conjuguant progrès technique construit sur une recherche finalisée, évolution des systèmes de production et conquête de marchés à l'export. En Indonésie, sur les traces du Vietnam, l'élevage de ces poissons-chats se développe également depuis quelques années en vue d'alimenter les marchés domestiques et l'exportion. L'élevage des "pangas " au Vietnam est une activité traditionnelle. Pour sécuriser un système basé sur la capture d'alevins et de juvéniles dans le milieu naturel, un programme scientifique franco-vietnamien a été mis en place en 1993, visant à maîtriser la reproduction artificielle des deux principales espèces de pangas d'élevage, Pangasius bocourti (élevé en cages flottantes) et Pangasianodon hypophthalmus (élevé en étangs fertilisés). Obtenue avec succès en 1995, elle entraîna une profonde mutation de la filière. L'espèce utilisée pour les élevages en cages et destinée à l'exportation, $P$. bocourti, a révélé la difficulté de mener à terme sa maturation sexuelle en captivité et sa relativement faible fécondité. La seconde espèce a démontré, quant à elle, une bonne aptitude à la maturation en captivité et une fécondité dix fois supérieure. $P$. hypophthalmus est ainsi devenue la principale espèce d'élevage. Dans le même temps, l'évolution s'est faite vers une utilisation croissante de l'aliment industriel du fait d'une raréfaction du poisson de rebut, ingrédient stratégique de l'aliment artisanal vietnamien. Les coûts de production sont de 0,6-0,7 US\$/kg (0,4-0,47 euro/kg) en moyenne. En Indonésie, l'élevage des pangas n'a véritablement démarré que dans les années 1990. Il est basé sur l'utilisation d'une espèce introduite, $P$. hypophthalmus, et d'une espèce locale, Pangasius djambal, élevées en étangs et en cages flottantes, en lacs ou en rivières, dans des exploitations le plus souvent familiales sur les îles de Java, Sumatra et Bornéo. Le Vietnam évalue sa production à 800000 t en 2006 et prévoit une production de 1000000 t en 2007, en quasi totalité exportée. En Indonésie, la production reste plus modeste mais significative, avec 31000 t en 2006.

Mots clés : Indonésie ; Pangasiidae ; pisciculture ; Vietnam.

Thèmes : économie et développement rural ; pêche et aquaculture ; productions animales.

\section{Abstract \\ Fish farming of Pangasiids}

For the last 15 years South East Asian fish farming of Pangasiids has shown dramatic growth and expansion. A main contributor in this field, Vietnam is the perfect example of a country having displayed a dynamics combining technical improvement derived from development oriented research, adaptability of culture systems and targeting of international markets. In Indonesia, following the example of Vietnam, fish farming of pangas catfish is developing too since a few years for both domestic and international markets. Fish farming of "pangas" is a traditional activity in Vietnam. In order to allow fish farmers not to depend on wild fry in stocking their ponds and cages, a collaborative scientific programme between France and Vietnam was set up in 1993 aimed at mastering artificial propagation of the two main cultured species, Pangasius bocourti (cultured in cages) and Pangasianodon hypophthalmus (cultured in manured ponds). Successfully achieved in 1995, the artificial propagation of pangas led to deep changes in the panga chain. Pangasius bocourti, grown in cages and the main species for export, displayed difficult sexual maturation in captivity and relatively low fecundity. The other species, $P$. hypophthalmus, showed easy maturation in captivity and a 10 times higher fecundity. Under these conditions, P. hypophthalmus has become the main culture spe- 
cies. At the same time, the trend evolved towards an increasing use of industrial feed due to the increasing price of trash fish used for on-farm feed manufacturing. Production costs come to 0.6-0.7 US\$ $/ \mathrm{kg}(0,4-0,47$ euro $/ \mathrm{kg})$. In Indonesia, fish farming of pangas really started in the nineties. It is based upon one exotic introduced species, $P$. hypophthamus, and an indigenous one, Pangasius djambal, cultured both in ponds and in cages, in lakes and in rivers, within mostly small scale family farms on Java, Sumatra and Borneo Islands. Vietnam's panga production is estimated at 800,000 $\mathrm{mt}$ for 2006 and $1 \mathrm{M} \mathrm{mt}$ for 2007, mainly exported. In Indonesia, production for 2006 is estimated at $31,000 \mathrm{mt}$.

Key words: fish culture; Indonesia; Pangasiidae; Vietnam.

Subjects: animal productions; economy and rural development; fishing and aquaculture.

a famille des Pangasiidae compte trois genres et 28 espèces réparties du Pakistan (Indus) à l'Indonésie. Le fleuve Mékong en compte à lui seul 14 et l'Indonésie 13, réparties dans les cours d'eau des îles de Java, Sumatra et Bornéo. Dans cette famille, les poissons d'intérêt aquacole correspondent à plusieurs espèces appartenant à deux genres distincts, Pangasius et Pangasianodon (Gustiano, 2003), regroupés sous l'appellation commerciale commune de "pangas".

La pisciculture des pangas est traditionnellement pratiquée dans les pays riverains du fleuve Mékong dans des cages flottantes en bambou dans lesquelles ils sont nourris à partir de sous-produits domestiques ou agricoles et dans des étangs le plus généralement fertilisés avec des effluents organiques (ICCILMB, 1992 ; Lazard et Legendre, 1993 ; Lazard et Cacot, 1997). Au début des années 1990, on estimait la production aquacole de pangas à environ 20 000-30000 t au Vietnam, 5 à $10000 \mathrm{t}$ au Cambodge et 15000 t en Thailande (Csavas, 1994). Au Vietnam, en l'absence de maîtrise de la reproduction en captivité, les alevins et juvéniles des espèces élevées étaient capturés dans le milieu naturel, principalement au Cambodge (> 80 \%). Des reproductions en captivité de Pangasius sp. avaient été réalisées dès la fin des années 1950 en Thailande (Boonbrahm, 1959) et ultérieurement au Vietnam (Huy et al., 1990 ; Kiem, 1992), mais avec une fiabilité insuffisante et un succès trop aléatoire pour asseoir un approvisionnement des structures d'élevage en alevins nés en captivité.

En Indonésie, jusqu'à un passé récent, hormis le grossissement en cages flottantes de juvéniles capturés par les pêcheurs en milieu naturel, peu de tentatives d'éle- vage avaient été réalisées avec les espèces locales de pangas. L'élevage de ces poissons-chats a véritablement commencé au début des années 1990, suite à l'introduction d'une espèce allochtone, Pangasianodon bypophthalmus (synonyme de Pangasius sutchi et de Pangasius hypophthalmus), dont la reproduction induite a été obtenue dans ce pays au début des années 1980 (Hardjamulia et al., 1981). Il s'est ensuite diversifié avec l'examen, puis la valorisation du potentiel aquacole des espèces locales de Pangasius.

Cet article est consacré à l'étude de la pisciculture des pangas au Vietnam et en Indonésie du fait de l'implication de ses auteurs dans ces deux pays.

\section{Espèces d'élevage}

Au Vietnam, parmi les 14 espèces de Pangasiidae inventoriées, les deux espèces les plus couramment élevées étaient, au début des années 1990, Pangasius bocourti et Pangasius bypophthalmus (Lenormand, 1996 ; Cacot, 1999). Chacune des deux espèces correspondait à un système d'élevage particulier en raison de leurs caractéristiques biologiques spécifiques.

$P$. bocourti est une espèce rhéophile (= espèce d'eau courante) particulièrement bien adaptée à l'élevage en cages flottantes sur le Mékong. Son régime alimentaire dans le milieu naturel est omnivore : végétaux (durant la crue), mollusques et crustacés (durant la saison sèche) (MRC, 2004). Elle présente une excellente efficacité d'utilisation de l'amidon qui permet, en élevage, de limiter les apports en protéines (figure 1) mais qui entraîne souvent un engraissement excessif (néolipogenèse) en élevage avec une alimentation riche en amidon (son de riz). La chair blanche et tendre est prisée sur les marchés locaux. Mais en fait, depuis la fin des années 1980, $P$. bocourti est également l'objet d'exportations sous forme de filets congelés malgré un faible rendement de filetage de l'ordre de $28 \%$, légèrement moins élevé que celui de $P$. hypophthalmus (30-32\%). Au Vietnam, l'élevage de cette première espèce était exclusivement pratiqué en cages flottantes au début des années 1990. Dans ces structures d'élevage, $P$. bocourti était fréquemment associé à deux petits cyprinidés : Leptobarbus hoevenii et Puntius altus; il pouvait également être associé à $P$. hypophthalmus. Son élevage en étangs est possible, mais il exige des conditions de qualité de l'eau beaucoup plus strictes (concentration en oxygène dissous, turbidité, composés azotés, etc.) et des densités de mise en charge moindres que $P$. hypophthalmus. P. hypophthalmus (figure 2) se caractérise, quant à lui, par une très grande tolérance vis-à-vis des faibles teneurs en oxygène dissous grâce à une vessie natatoire richement vascularisée qui lui permet d'utiliser l'oxygène atmosphérique. Cette caractéristique fait de cette espèce un excellent candidat à l'élevage en étangs à forte densité et faible renouvellement d'eau (ou même en eau stagnante) et fortement enrichi en matière organique. Son régime alimentaire dans le milieu naturel est très voisin de celui de $P$. bocourti avec une tendance détritivore marquée. Globalement moins apprécié sur le marché local vietnamien, de même que sur les autres marchés de la région, du fait d'une qualité de chair aléatoire liée aux conditions d'élevage (couleur jaunâtre, texture, goût de vase), $P$. bypoph- 


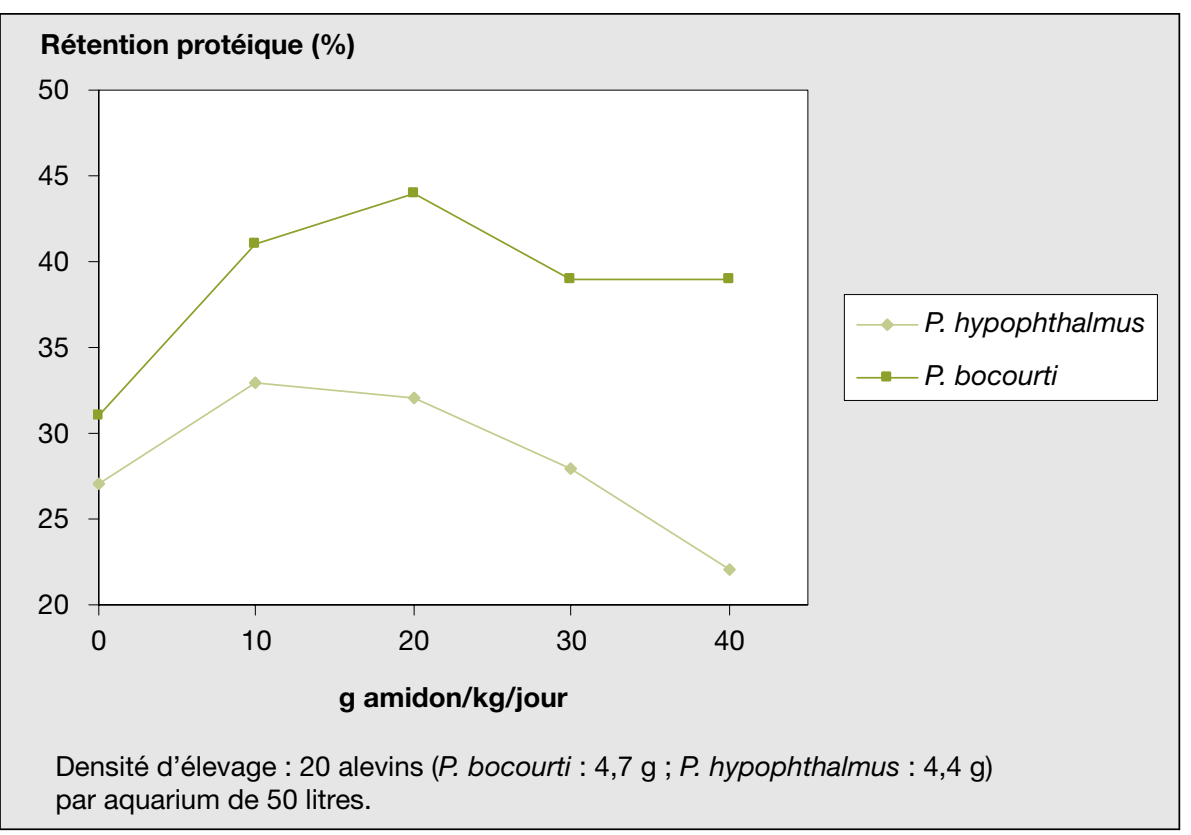

Figure 1. Effet de l'incorporation croissante d'amidon dans la ration alimentaire de Pangasianodon hypophthalmus et Pangasius bocourti (apport constant en protéines: $15 \mathrm{~g}$ de protéines/kg par jour) durant 28 jours d'après Hung et al., 2003.

Figure 1. Crude protein gain as a function of diet corresponding to increasing daily starch allowance with the same daily protein allowance (15 g protein $/ \mathrm{kg}$ per day) over 28 days in Pangasianodon hypophthalmus and Pangasius bocourti after Hung et al., 2003.
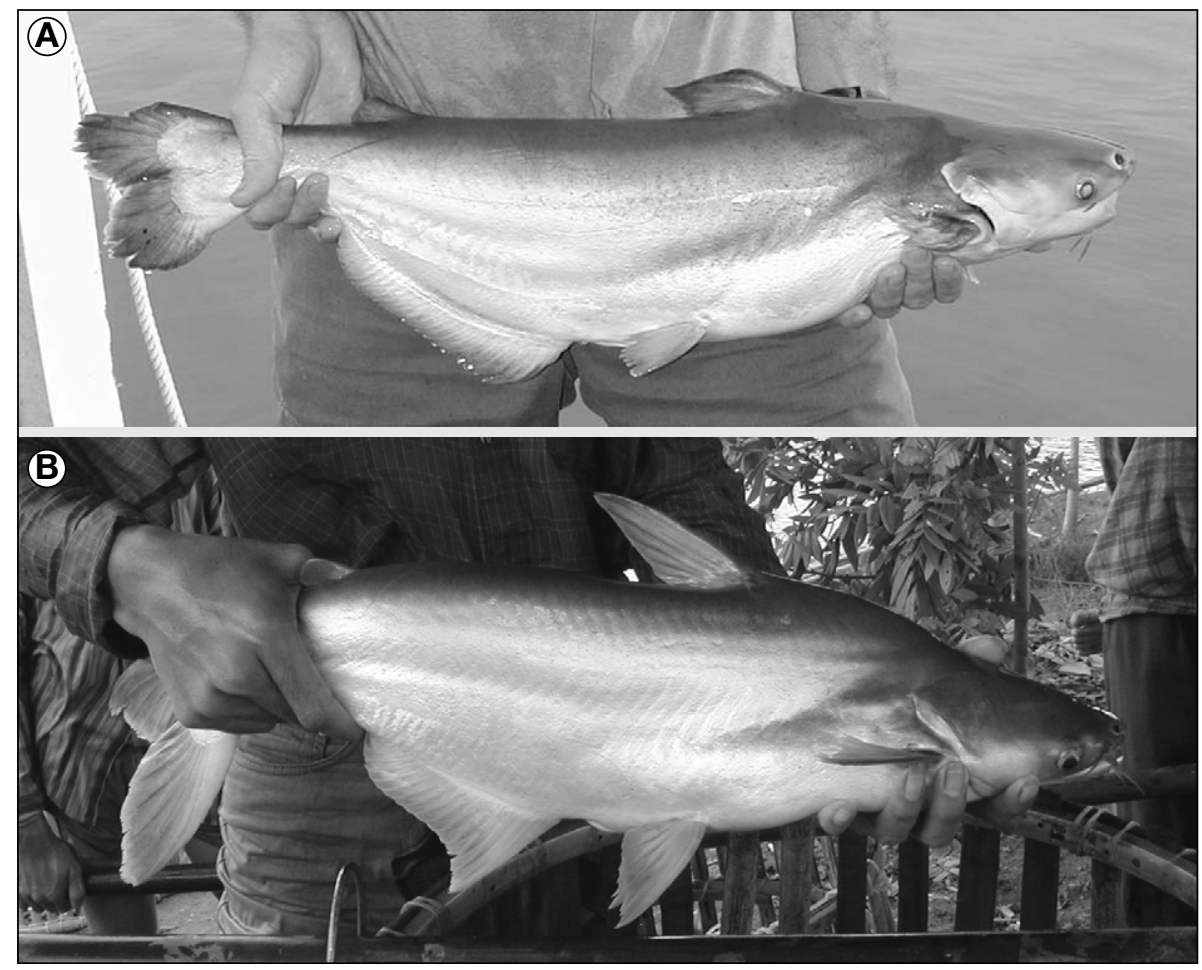

Figure 2. Pangasius bocourti (A) et Pangasianodon hypophthalmus (B) [photos P. Cacot].

Figure 2. Pangasius bocourti (A) et Pangasianodon hypophthalmus (B) [photos P. Cacot]. thalmus domine cependant aujourd'hui très largement la pangasiculture vietnamienne, du fait notamment de sa fécondité élevée et de son meilleur rendement au filetage, et alimente aussi bien le marché d'export que les marchés locaux.

En Indonésie, bien que la pisciculture en eau douce soit une pratique ancestrale sur lîle de Java et que 13 espèces de Pangasiidae soient présentes dans les eaux continentales de ce pays, l'élevage des pangas se limitait jusque dans les années 1990 au stockage de poissons en cages flottantes pendant la saison de pêche pour les revendre, hors saison, à meilleur prix. Ces pratiques restaient d'ailleurs assez exceptionnelles et très localisées sur les îles de Sumatra et de Bornéo, où ces poissons-chats sont plus appréciés par les consommateurs qu'ils ne le sont à Java. Jusqu'à la fin des années 1990, la seule espèce de pangas d'élevage était une espèce introduite à partir de la Thailande en 1972, P. hypophthalmus, présentée cidessus. Cette espèce, destinée dans un premier temps à l'industrie des poissons d'ornement, a été cultivée rapidement comme espèce de consommation commercialisée sur les marchés locaux. Les premières écloseries ainsi que les premières fermes de grossissement ont vu le jour dans la région de Bogor dans la province de Java-Ouest, et ce n'est qu'une quinzaine d'années plus tard que l'élevage de $P$. hypophthalmus s'est vraiment développé sur les îles de Sumatra et de Bornéo, à partir d'alevins produits par les écloseries de Bogor. Par la suite, l'examen approfondi des potentialités aquacoles des Pangasiidae autochtones a abouti à la mise en évidence des caractéristiques zootechniques favorables de Pangasius djambal, puis à la maîtrise de son cycle d'élevage après avoir constitué des stocks de géniteurs à partir d'individus sauvages (Legendre et al., 2000a ; Slembrouck et al., 2003).

$P$. djambal a été choisi comme espèce d'élevage du fait de sa forte valeur marchande, de sa taille maximale élevée (plus d'un mètre) et de sa large répartition géographique. Ce panga vit dans les cours d'eau des îles de Sumatra, Bornéo et Java, et comme $P$. bocourti dont il est phylogénétiquement proche (Pouyaud et al., 2000), c'est une espèce rhéophile et omnivore. Elle est bien adaptée à l'élevage en cages flottantes et en étangs, où elle fait preuve d'une grande robustesse et présente une croissance supérieure à celle de $P$. hypophthalmus (Legendre et al., 2000a). Elle est, cependant, plus sen- 
sible que ce dernier à de faibles concentrations en oxygène dissous. Outre son appréciation par les consommateurs locaux, sa chair blanche et dépourvue d'arêtes convient parfaitement à une production tournée vers l'exportation de filets congelés.

\section{Maîtrise}

\section{de la reproduction et conséquences}

\section{Maturation sexuelle et reproduction induite en captivité}

Comme la plupart des poissons, les pangas ne se reproduisent pas spontanément en captivité et un traitement hormonal est nécessaire pour la stimulation de la spermiation et l'induction de l'ovulation.

$\mathrm{Au}$ Vietnam, la première reproduction en captivité de $P$. bocourti a été réalisée en mai 1995 (Cacot et al., 2002 ; Cacot et al., 2003). Appliquée à l'autre espèce, P. bypophthalmus, elle a totalement "révolutionné " la filière en levant son principal verrou: l'approvisionnement contrôlé en alevins et juvéniles. Celui-ci était devenu problématique à la fois sur le plan politique (durcissement des relations entre le Cambodge et le Vietnam, notamment sur le sujet très sensible des ressources halieutiques) et économique (l'envolée du prix des alevins et juvéniles de pangas était telle que ce poste représentait, en 1995, jusqu'à 52 \% des coûts de production de $P$. bocourti en cages flottantes ; Cacot et Lazard, 2004).

Les géniteurs utilisés pour ces premières reproductions étaient entretenus depuis plusieurs années, en cages par l'entreprise AGIFISH ( $P$. bocourti et $P$. bypophthalmus) et en étangs par l'université de Can Tho (P. bypophthalmus). Sans connaitre leur âge avec précision, on peut l'estimer en moyenne pour l'ensemble des géniteurs à environ quatre à cinq ans (poids moyen de $5 \mathrm{~kg}$ ). L'échec de toutes les tentatives de reproduction artificielle de $P$. bocourti s'explique principalement du fait d'une maturation gonadique incomplète en captivité qui ne permet pas d'obtenir la maturation ovocytaire et l'ovulation après un traitement hormonal classique. La technique utilisée pour surmonter cet obstacle fut de recourir à une série d'injections d'hormone gonadotrope, en l'occurrence l'hCG (buman chorionic gonadotropin) administrée à faible dose, de façon répétée et quotidienne, durant six à sept jours en moyenne (Cacot et al., 2002). Ce traitement préparatoire, qui permet d'accroître et d'homogénéiser le diamètre des ovocytes postvitellogéniques, est ensuite suivi d'un traitement plus conventionnel constitué de deux injections successives, l'une de priming et l'autre d'induction de la maturation ovocytaire et de l'ovulation (figure 3). P. bypophthalmus, quant à lui, achève complètement sa maturation sexuelle en captivité, et deux injections successives sont suffisantes pour induire l'ovulation (d'où les succès plus rapides obtenus sur cette espèce). Les mâles des deux espèces ne posent pas de problème particulier et sont fluents en saison de reproduction. Une seule injection leur est appliquée afin d'augmenter le volume de laitance émis et de faciliter la collecte du sperme.

En Indonésie, à la suite de la première reproduction induite de $P$. hypophthalmus, les techniques ont ensuite été opti- misées pour cette espèce par des traitements hormonaux plus appropriés avec une attention particulière portée au respect du temps de latence entre traitement hormonal et collecte des ovules (Legendre et al., 2000b). Chez ce poisson, la qualité des ovules ne se maintient pas au-delà d'un délai de deux à trois heures après l'ovulation, et un mauvais respect du temps de latence explique dans une large mesure les mauvais résultats souvent observés dans les piscicultures. Bien que la maturité sexuelle des géniteurs de $P$. bypophthalmus maintenus en étangs présente des variations saisonnières, des femelles et des mâles matures sont présents en permanence dans les stocks d'élevage, permettant une production de larves tout au long de l'année (Legendre et al., 1999; Kristanto et al., 2005). Au Vietnam, la saisonnalité de la reproduction de cette espèce est cependant beaucoup plus marquée, et des pontes ne peuvent être obtenues que durant une période limitée à quelques mois durant la saison des pluies (Cacot, 1999). Contrairement à la situation observée chez $P$. bocourti, $P$. djambal parvient à pleine maturité sexuelle lorsqu'il est

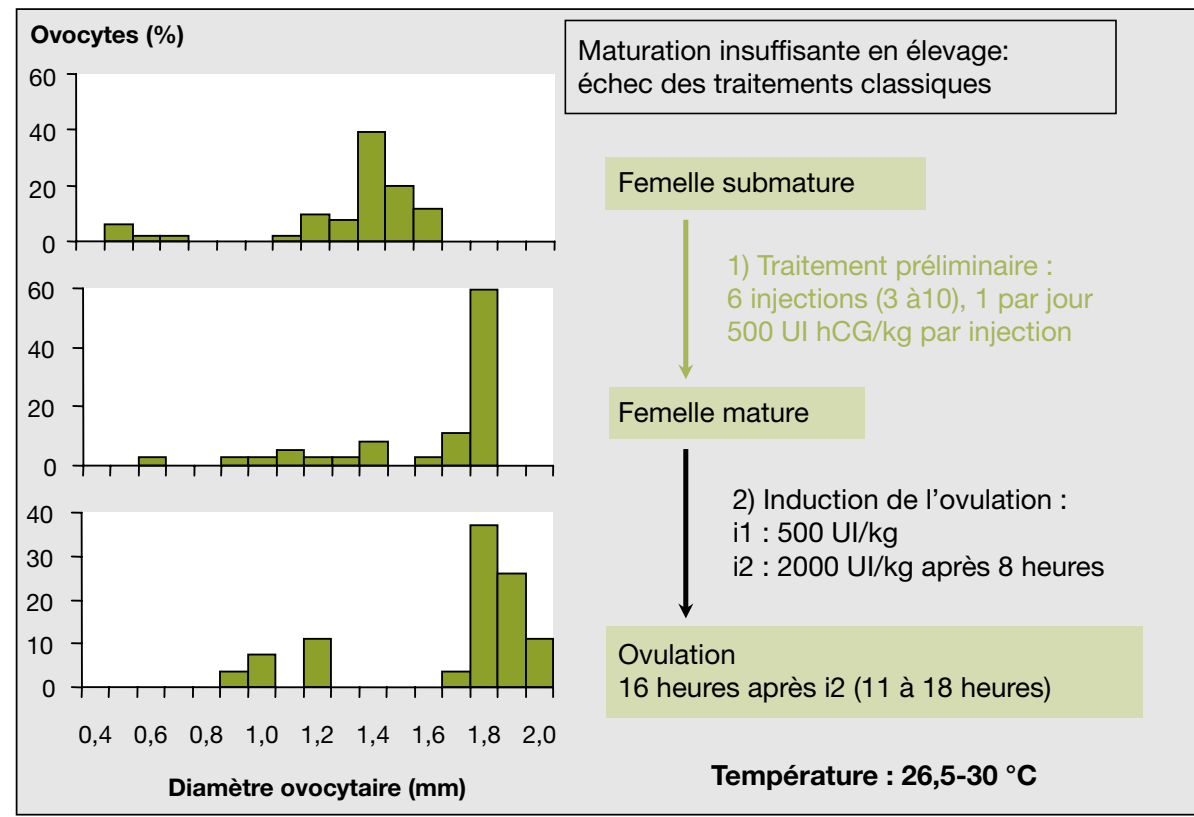

Figure 3. Achèvement de la maturation gonadique avant induction de la maturation ovocytaire et de I'ovulation chez Pangasius bocourti par recours à des traitements préliminaires d'human chorionic gonadotropin, hCG (Cacot et al., 2002).

Figure 3. Achievement of gonadal maturity before induction of oocyte maturation and ovulation in Pangasius bocourti using hCG priming treatment (Cacot et al., 2002).

Remarque : dans le troisième histogramme, les ovules d'un diamètre compris entre 1,6 et 1,8 mm correspondent aux ovules collectés par stripping.

$\mathrm{UI}$ : unité internationale. 
élevé en étangs ou en cages flottantes. De ce fait, un traitement hormonal constitué de deux injections successives, de hCG puis de Ovaprim à 24 heures d'intervalle (priming puis dose ovulatoire (Slembrouck et al., 2003)), permet d'induire la maturation ovocytaire et l'ovulation chez plus de $80 \%$ des femelles traitées avec une très bonne qualité des ovules obtenus.

En aval de la reproduction en captivité l'élevage larvaire de ces espèces a fait l'objet de nombreux travaux expérimentaux (Hung et al., 1999; Subagja et al., 1999 ; Slembrouck et al., 1999; Hung, 2000 ; Legendre et al., 2000a; Hung et al., 2001; Hung et al., 2002 ; Hung et al., 2003; Slembrouck et al., 2003). Les réserves vitellines plus importantes et l'absence de comportement cannibale des larves chez $P$. bocourti (et chez $P$. djambal) permettent son alevinage en structures intensives à fortes densités, dont les performances avec différents types d'aliments (vivants et inertes) sont données dans la figure 4. Pour P. bypophthalmus, les nombreux essais réalisés en structures intensives, avec alimentation à base de proies vivantes, se sont longtemps révélés peu fructueux avec des taux de mortalité observés généralement supérieurs à $90 \%$ du fait du comportement cannibale fréquemment rapporté chez cette espèce. Face à ces contraintes, la stratégie adoptée par les pisciculteurs vietnamiens a consisté en un alevinage en petits étangs fertilisés et aérés à partir de j1 (un jour postéclosion) avec alimentation à base de jaune d'œuf et de levure les premiers jours, suivis d'une alimentation naturelle à partir de zooplancton, puis d'un complément avec un aliment artificiel riche en protéines. En Indonésie, les travaux effectués sur l'élevage larvaire de cette espèce ont toutefois montré que les infections bactériennes étaient responsables d'une mortalité plus élevée des larves que celle résultant directement du cannibalisme (Subagja et al., 1999). Des traitements antibactériens de l'eau (antibiotiques ou désinfectants) dans des élevages en circuit fermé ont permis d'améliorer très substantiellement les taux de survie moyens des larves en fin d'élevage larvaire (jusqu'à plus de $86 \%$ à l'âge de 10 jours).

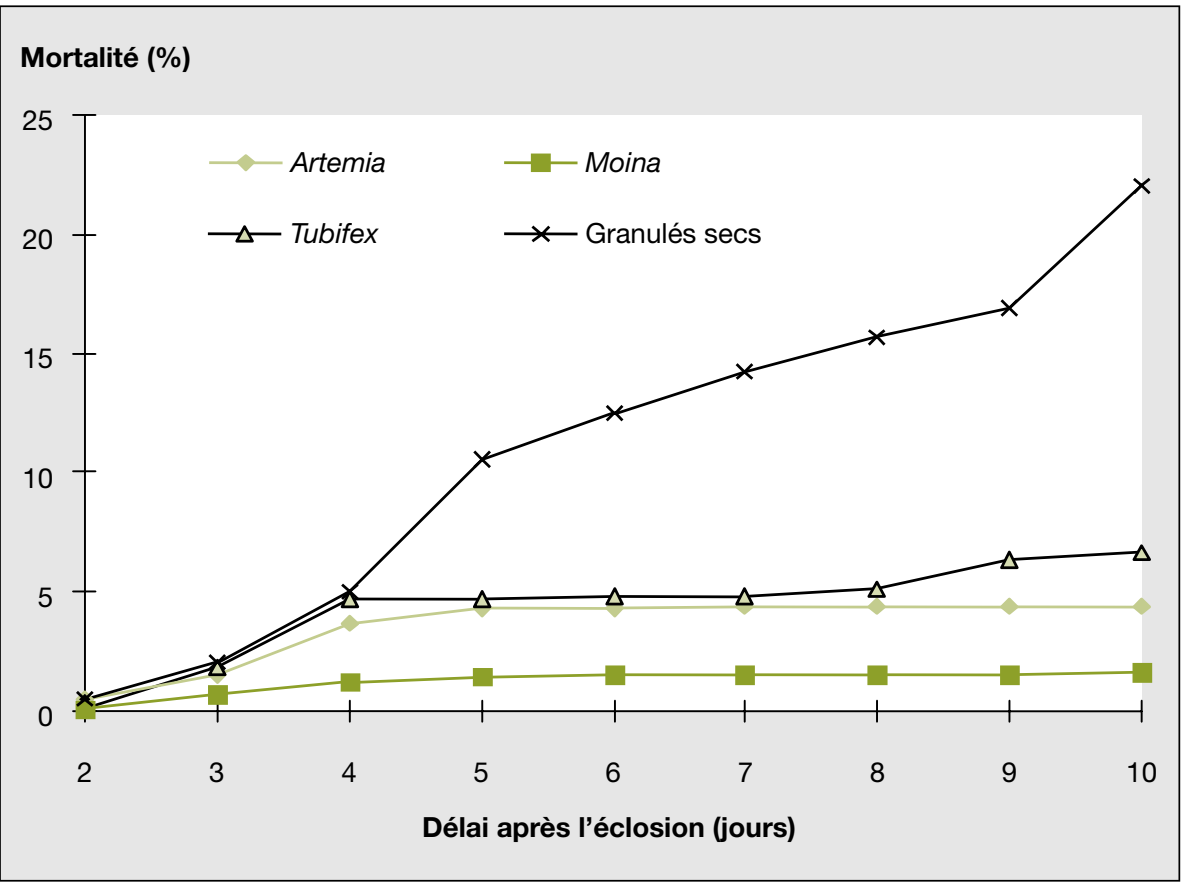

Figure 4. Évolution de la mortalité de larves de Pangasius bocourti élevées à forte densité (500 larves/ aquarium de 50 litres) à partir de j2 (deux jours après éclosion) en structures intensives (aquariums et bacs) soumises à différents régimes alimentaires (proies vivantes et aliment démarrage truite) d'après Hung et al., 2002.

Figure 4. Cumulative mortality of Pangasius bocourti larvae in response to live feed or trout feed starter at high density (500 larvae/50-liter aquariums). Feeding started 48 hours after hatching (D2) after Hung et al. 2002.

\section{De la découverte à l'innovation}

La technicité, le dynamisme et l'esprit d'entreprise des acteurs vietnamiens de la filière piscicole du delta du Mékong ont transformé la découverte de la mâ̂trise de la reproduction en captivité des deux espèces de Pangasiidae en une innovation que s'est appropriée une nouvelle catégorie d'opérateurs aquacoles: plusieurs centaines d'écloseries ont vu le jour dans le delta et sont désormais en mesure d'approvisionner intégralement les élevages en cages et en étangs, pour le marché de l'export et le marché local (Anonyme, 2001 ; Huillery, 2001 ; Freud et Richard, 2002).

Sur le plan des espèces, une profonde mutation est intervenue également. La reproduction en captivité a fait apparaître de profondes différences de caractéristiques biologiques entre les deux espèces. $P$. bocourti, initialement la plus recherchée pour l'exportation, a révélé trois handicaps majeurs pour une production massive d'alevins en conditions contrôlées : maturation complète difficile en captivité nécessitant un traitement hormonal de "finition", faible fécondité (7 500 œufs par kilogramme de femelle ; figure 5) et élevage larvaire inféodé à des structures intensives avec première alimentation à base de proies vivantes assurant une survie élevée rendue nécessaire du fait de sa faible fécondité (Hung et al., 2002). P. hypophthalmus, sur ces trois points, s'est révélé beaucoup plus performant: bonne maturation en captivité, fécondité élevée (> 80000 œufs et jusqu'à 200 000/kg de femelle ; figure 5), survie larvaire économiquement acceptable (30$70 \%$ d'après Huillery, 2001 et Hung, données non publiées) en étangs extensifs fertilisés malgré la petite taille des oufs. Ces caractéristiques biologiques ont induit, en quelques années, une totale substitution de la principale espèce d'élevage en cages flottantes destinée à l'exportation : $P$. hypophthalmus a remplacé $P$. bocourti et constitue aujourd'hui plus de $95 \%$ des pangas produits et l'essentiel de ceux exportés ; P. bocourti n'est plus élevé que marginalement. La couleur de la chair et le goût de vase de $P$. hypophthalmus élevé en structures extensives, liés à la consommation par ce poisson d'importantes quantités de phytoplancton riche en pigments et en géosmine, ont été radicalement modifiés : chair blanche et absence de goût de vase lorsqu'il est élevé en conditions intensives 


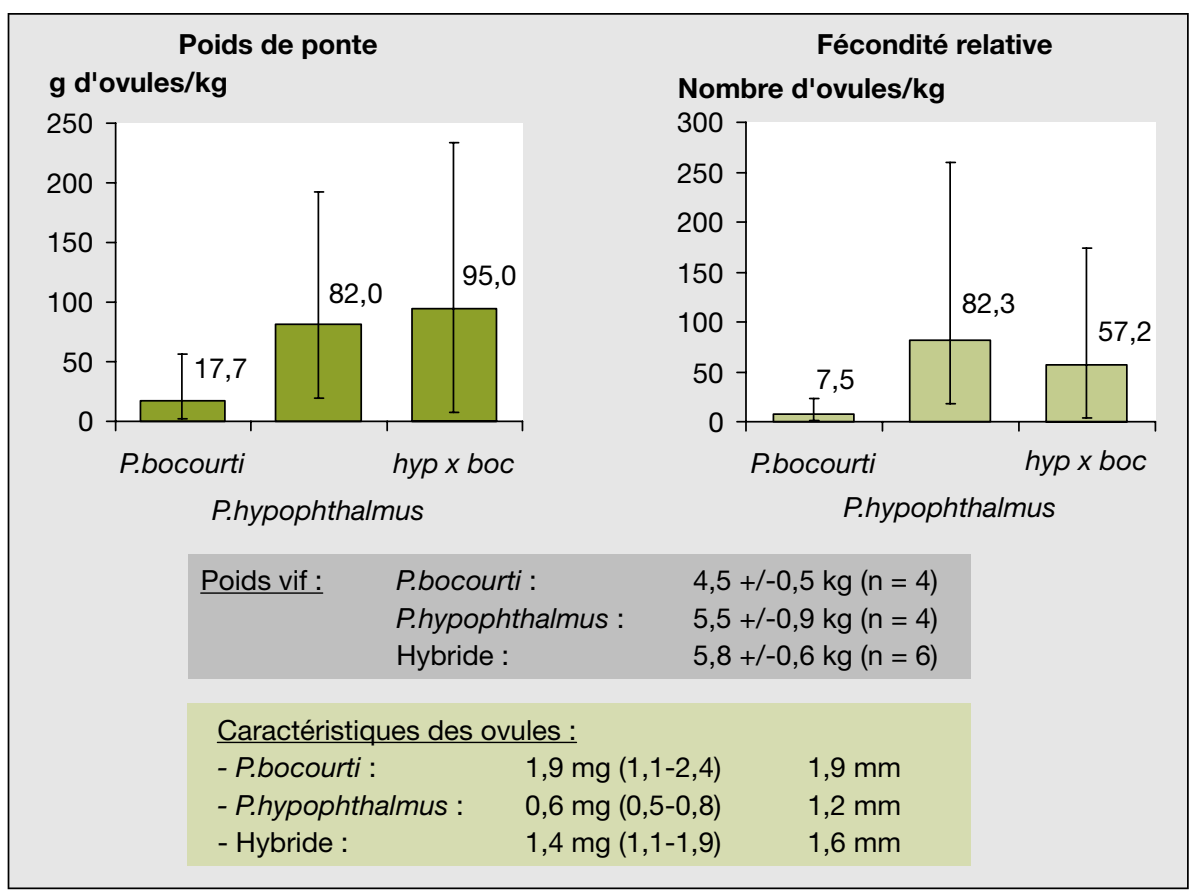

Figure 5. Fécondité relative et caractéristique des géniteurs de Pangasius bocourti, Pangasianodon hypophthalmus et de leur hybride (P. b. $\sigma^{x} \times$ P.h. O) dans le delta du Mékong. (Campet, données non publiées).

Figure 5. Relative fecundity and characteristics of breeders from Pangasius bocourti, Pangasianodon hypophthalmus and their hybrid (P. b. $\sigma^{\pi} \times$ P.h. O) in the Mekong delta (Campet, unpublished data). $\mathrm{n}$ : nombre de sujets.

à fort renouvellement d'eau (cages, enclos, étangs).

En 2006, on estimait la production totale de Pangasius à 800000 t, dont $700000 \mathrm{t}$ destinées à l'exportation. Celle-ci est effectuée en totalité sous forme de filets congelés produits dans 84 usines toutes localisées dans le delta du Mékong.

En Indonésie, l'élevage larvaire de $P$. hypophthalmus est le plus souvent réalisé à une densité d'empoissonnement de 30 à 50 larves par litre en aquariums en eau stagnante renouvelée quotidiennement. Les larves sont alimentées avec des nauplii d'Artemia durant les deux premiers jours d'alimentation exogène, puis avec Tubifex et des vers de vase pendant les deux semaines suivantes. Les alevins sont ensuite prégrossis en aquarium ou en étangs (jusqu'à une taille d'environ $5 \mathrm{~cm}$ ), avant d'être disséminés au niveau des fermes de grossissement où l'élevage est poursuivi en étangs ou en cages flottantes. Les exploitations sont de tailles variées mais souvent modestes et de type familial.

Depuis quelques années, grâce à la mâ̂trise de l'élevage de $P$. djambal et à l'incitation du gouvernement, l'élevage des Pangasiidae tend à se diversifier en Indo- nésie. Bien que $P$. djambal soit phylogénétiquement proche de $P$. bocourti, sa fécondité (en moyenne $12500 \pm 5500$ ovules $/ \mathrm{kg}$ de femelle) est plus élevée que celle de $P$. bocourti pour une taille d'ovule similaire (environ 1,8 mm de diamètre). Néanmoins, la fécondité de $P$. djambal, encore en retrait par rapport à celle de $P$. hypophthalmus, reste souvent considérée comme un handicap au niveau des écloseries, en dépit d'un élevage larvaire beaucoup plus simple à mầtriser du fait de la plus grande taille des larves à leur entrée en phase trophique. Comme cela avait été le cas au Vietnam à la fin des années 1990 entre $P$. hypophthalmus et $P$. bocourti (figure 5), ce constat a conduit les acteurs de la filière pangas en Indonésie à envisager l'utilisation d'un hybride entre $P$. hypophthalmus et $P$. djambal qui permettrait de combiner la forte fécondité du premier avec la bonne survie larvaire, les caractéristiques de croissance et la qualité de chair du second. On sait cependant que cet hybride n'est pas stérile, et son échappement possible à partir des structures d'élevage représente donc un risque de contamination génétique pour les populations naturelles (Anonyme, 2000 ).
En 2006, la production de pangas était estimée à 31000 t en Indonésie, provenant environ pour moitié des élevages en étangs et pour moitié des élevages en cages flottantes en rivière ou en réservoir.

\section{Évolution}

\section{des structures d'élevage et de l'alimentation}

Au Vietnam, initialement réalisé exclusivement en cages flottantes, l'élevage de Pangasius destiné à l'exportation, en même temps qu'il changeait d'espèce, évoluait vers des systèmes dont les infrastructures (étangs et enclos) étaient moins coûteuses du fait de la hausse considérable du prix du bois nécessaire à la construction des cages. C'est ainsi que ces trois types d'infrastructures contribuaient pour 22 , 13 et $65 \%$ respectivement pour les cages, les enclos et les étangs à la production totale en 2006.

À l'origine, entièrement basé sur l'utilisation d'un aliment artisanal fabriqué sur la cage d'élevage (farm-made aquafeed), l'élevage évolue vers une utilisation croissante d'aliment industriel du fait d'une raréfaction (et donc d'une augmentation du coût) du poisson de rebut (trash fish), ingrédient stratégique de l'aliment artisanal qui en renferme $40 \%$ mélangés à $60 \%$ de son de riz. L'aliment industriel utilisé pour la phase de grossissement de $P$. hypophthalmus (200 g à $1,2 \mathrm{~kg}$ ) contient entre 27 et $22 \%$ de protéines quasiment, entièrement, d'origine végétale (tableau 1). En outre, les travaux réalisés par Hung et al. (2004) sur des alevins des trois espèces de pangas étudiées dans cet article révèlent la meilleure croissance spécifique de $P$. bocourti, suivi de $P$. djambal, puis de $P$. hypophthalmus. Par ailleurs, le taux le plus élevé de rétention protéique est observé chez $P$. djambal et le taux de dépôt lipidique le plus élevé, chez $P$. bocourti.

Les rendements en poisson marchand, au Vietnam, révèlent des rendements extrêmement élevés pour les infrastructures du type de celles utilisées : ils varient entre 200 et 600 t/ha par an pour les étangs en fonction du taux de renouvellement d'eau, $1000 \mathrm{t}$ pour les enclos et $200 \mathrm{~kg} / \mathrm{m}^{3}$ par an pour les cages flottantes (tableau 2). 


\section{Tableau 1. Composition moyenne d'un aliment industriel pour l'élevage intensif de Pangasianodon hypophthalmus en étangs au Sud-Vietnam.}

Table 1. Nutrient composition of an average commercial formulated feed for intensive Pangasianodon hypophthalmus culture in pond in South Vietnam.

\begin{tabular}{|c|c|c|c|c|c|c|c|c|}
\hline \multirow{3}{*}{$\begin{array}{l}\text { Présentation/taille }(\mathrm{mm}) \\
\text { Phase d'élevage } \\
\text { Nutriments }\end{array}$} & \multirow{2}{*}{\multicolumn{2}{|c|}{$\begin{array}{c}\text { Extrudé 1,5 } \\
\begin{array}{c}5-20 \mathrm{~g} \\
\text { (nurserie) }\end{array}\end{array}$}} & \multirow{2}{*}{\multicolumn{2}{|c|}{$\begin{array}{c}\text { Extrudé } 2-3 \\
20-200 \mathrm{~g} \\
\text { régrossissement) }\end{array}$}} & \multirow{2}{*}{\multicolumn{2}{|c|}{$\begin{array}{l}\text { Extrudé } 5 \\
200-400 \mathrm{~g} \\
\text { ossissement 1) }\end{array}$}} & \multirow{2}{*}{\multicolumn{2}{|c|}{$\begin{array}{c}\text { Extrudé 7-10 } \\
\begin{array}{c}400-1000 \mathrm{~g} \\
\text { (grossissement 2) }\end{array}\end{array}$}} \\
\hline & & & & & & & & \\
\hline & Minimum & Maximum & Minimum & Maximum & Minimum & Maximum & Minimum & Maximum \\
\hline $\begin{array}{l}\text { Énergie digestible calculée } \\
(\mathrm{kJ} / \mathrm{kg})\end{array}$ & 10965 & & 10546 & & 10253 & & 9458 & \\
\hline Humidité (\%) & & 12 & & 12 & & 12 & & 12 \\
\hline Protéine (\%) & 30 & & 28 & & 27 & & 22 & \\
\hline Matières grasses (\%) & 4,5 & 5,5 & 4,5 & 5,5 & 4,5 & 5,5 & 4,5 & 5,5 \\
\hline Matières minérales (\%) & & 12 & & 12 & & 12 & & 12 \\
\hline Cellulose (\%) & & 6 & & 6 & & 6 & & 5,6 \\
\hline Amidon (\%) & 19 & 30 & 19 & 30 & 19 & 30 & 19 & 30 \\
\hline Calcium (g/kg) & 9 & 21 & 9 & 20 & 9 & 20 & 11 & 20 \\
\hline Phosphore (g/kg) & & 15,5 & & 15,5 & & 15,5 & & 15,5 \\
\hline Sodium (\%) & 0,5 & 2 & 0,5 & 2 & 0,5 & 2 & 0,5 & 2 \\
\hline Chlore (\%) & & 2 & & 2 & & 2 & & 2 \\
\hline Lysine (\%) & 1,5 & & 1,4 & & 1,3 & & 1 & \\
\hline Méthonine (\%) & 0,7 & & 0,6 & & 0,5 & & 0,5 & \\
\hline
\end{tabular}

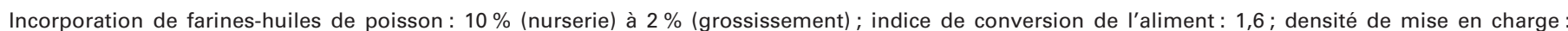
$33 / \mathrm{m}^{2}$; taux de survie : $70 \%$; durée du cycle d'élevage : 180 jours.

Les coûts de production varient selon les structures d'élevage et d'aliment utilisées : ils sont en moyenne de 0,6-0,7 US $\$ / \mathrm{kg}(0,40-0,47$ euro/kg). Le détail des comptes d'exploitations en étangs gérés avec les deux types d'aliments est donné dans le tableau 3. Le bilan des prix de revient selon les quatre traitements (deux types d'aliments/deux types d'infrastructures) est donné dans la figure 6 (Hung et al., 2006).

La question de l'utilisation d'antibiotiques dans la pangasiculture vietnamienne constitue aujourd'hui un véritable enjeu en termes de qualité. Il est très difficile de connaitre les substances actives des antibiotiques utilisés dans les élevages de pangas au Vietnam, mais des résultats récents, mettant en évidence des antibiorésistances de la flore bactérienne isolée à partir de poissons d'élevage, laissent supposer une mauvaise maîtrise de la gestion sanitaire des élevages (Sarter et al., 2006). L'achat des produits médicamenteux pour poissons constitue le troisième poste des coûts de production (>5\%). Cependant, le principe d'équivalence (règl. CE 178/2002 et règl. CE 852/2004) qui impose aux produits importés en Europe une qualité sanitaire au moins égale à celle des produits européens s'applique ici. Les mesures de contrôle mises en ouvre par les autorités vietnamiennes ont été habilitées par la CE.

L'exportation des filets de pangas du Vietnam, après avoir été principalement dirigée vers les États-Unis (fin des années 1990, début des années 2000), s'oriente actuellement vers les clients traditionnels d'Asie (Japon, Hong Kong, Singapour) et, de façon croissante, vers l'Europe (notamment de l'Est). Cette évolution s'explique par les très nombreuses barrières douanières érigées, par les autorités américaines vis-à-vis des poissons-chats du Mékong pour protéger leur propre marché de poisson-chat américain commercialisé sous l'appellation de catfish.

\section{Tableau 2. Caractéristiques zootechniques des élevages de Pangasianodon hypophthalmus dans le delta du Mékong (Guibert, 2003).}

Table 2. Zootechnical characteristics of Pangasianodon hypophthalmus culture systems in the Mekong delta (Guibert, 2003).

\begin{tabular}{|c|c|c|c|c|c|}
\hline & Nombre & $\begin{array}{l}\text { Densité } \\
\left(\mathrm{m}^{2} / \mathrm{m}^{3}\right)\end{array}$ & $\begin{array}{c}\text { Poids moyen } \\
\text { initial }(\mathrm{g})\end{array}$ & Rendement & $\begin{array}{c}\text { Mortalité } \\
(\%)\end{array}$ \\
\hline Étang avec faible renouvellement d'eau & 5 & 18 & 30 & 200-350 t/ha par an & - \\
\hline Étang avec renouvellement moyen d'eau & 4 & 25 & 12 & 500-600 t/ha par an & $4-25$ \\
\hline Étang avec fort renouvellement d'eau & 3 & $25-40$ & $5-100$ & $600-650$ t/ha par an & $10-15$ \\
\hline Enclos & 2 & $50-75$ & $15-40$ & > 1000 t/ha par an & 10 \\
\hline Cage & 4 & 110 & 100 & $14-17 \mathrm{~kg} / \mathrm{m}$ par mois & $10-30$ \\
\hline
\end{tabular}


Tableau 3. Coûts de production de Pangasianodon hypophthalmus en étangs (par $\mathbf{m}^{2}$ ) (Hung et al., 2006).

Table 3. Average production costs of Pangasianodon hypophthalmus in pond culture (per one square meter) (Hung et al., 2006).

\begin{tabular}{|c|c|c|c|c|}
\hline \multirow[t]{2}{*}{ Postes } & \multicolumn{2}{|c|}{ Aliment artisanal $(n=11)$} & \multicolumn{2}{|c|}{ Aliment industriel $(n=23)$} \\
\hline & Coûts ${ }^{a}$ & Pourcentage total & Coûts $^{a}$ & Pourcentage total \\
\hline \multicolumn{5}{|l|}{ Coûts fixes } \\
\hline $\begin{array}{l}\text { Amortissement (étang, } \\
\text { embarcation, unité alimentation) }\end{array}$ & $2394 \pm 2157$ & 0,80 & $3051 \pm 4179$ & 0,89 \\
\hline Location étang & - & - & $1073 \pm 646$ & 0,31 \\
\hline \multicolumn{5}{|l|}{ Coûts variables } \\
\hline Fingerlings & $29189 \pm 43950$ & 9,81 & $16480 \pm 12369$ & 4,82 \\
\hline Aliment & $241368 \pm 135601$ & 81,08 & $309759 \pm 193192$ & 90,62 \\
\hline Main d'œuvre & $2928 \pm 1420$ & 0,98 & $2813 \pm 1726$ & 0,82 \\
\hline Médicaments & $15999 \pm 9711$ & 5,37 & $8136 \pm 13639$ & 2,38 \\
\hline Fuel et électricité & $5495 \pm 2361$ & 5,37 & $1170 \pm 2429$ & 0,34 \\
\hline Intérêts d'emprunt & - & - & $1667 \pm 104$ & 0,49 \\
\hline Taxes & $130 \pm 89$ & 0,04 & $924 \pm 1635$ & 0,27 \\
\hline Transport & $179 \pm 243$ & 0,06 & $1364 \pm 695$ & 0,40 \\
\hline \multicolumn{5}{|l|}{ Coût total } \\
\hline Par mètre carré & $297682 \pm 170026$ & & $341820 \pm 10168$ & \\
\hline Par kilogramme de poisson & $8786 \pm 1836$ & & $9535 \pm 1535$ & \\
\hline
\end{tabular}

${ }^{a}$ Les coûts sont donnés en VN dongs (1 dollar US = 16500 VN dongs en moyenne en 2008).

\section{Coût de production par kilo de poisson (en VND)}

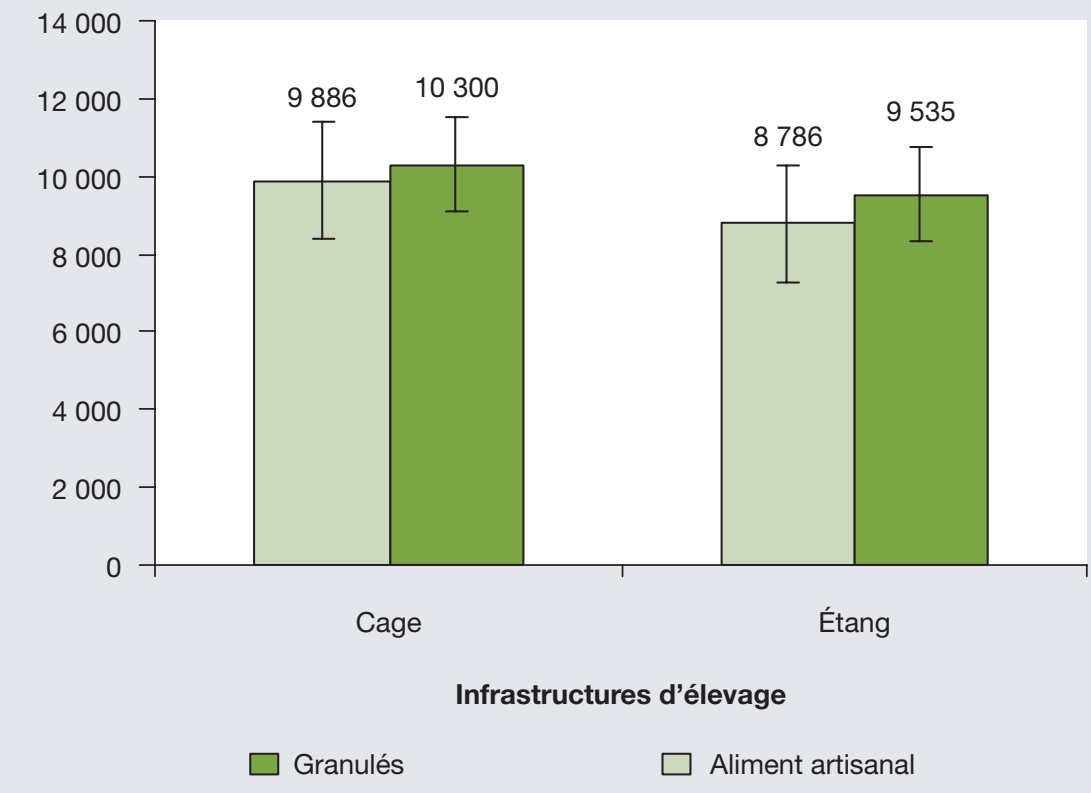

Figure 6. Coûts de production comparés de Pangasianodon hypophthalmus en cages et en étangs avec un aliment artisanal et un aliment composé industriel dans le delta du Mékong (Hung et al, 2006).

Figure 6. Comparative production costs of Pangasianodon hypophthalmus in cages and ponds using an on-farm manufactured feed and an industrial compounded feed in the Mekong delta (Hung et al., 2006).

VND : Dông vietnamien (1 dollar US = 16500 VND en 2008).
La composition moyenne des filets de $P$. bypophthalmus est de $82 \%$ d'eau, $15,5 \%$ de protéines et $2 \%$ de lipides (Guibert, 2003).

Une synthèse de la filière vietnamienne de pangas est présentée dans la figure 7. En Indonésie, l'augmentation régulière du coût des aliments commerciaux depuis quelques années et le surcoût associé en fonction de la distance séparant l'exploitation des fabriques d'aliments constituent une contrainte forte pour le développement de la filière. Une des conséquences est l'apparition récente dans les régions éloignées des centres de production, d'unités artisanales de fabrication d'aliments avec utilisation de rejets agricoles et agro-industriels locaux et incorporation de poissons de rebut en provenance des pêcheries maritimes les plus proches.

\section{Conclusion}

Les pangas offrent l'exemple d'un groupe d'espèces dont la domestication lancée récemment a permis le développement 
> 95 \% Pangasius hypophthalmus Production totale : 800000 tonnes - Étangs : $65 \%$

- Cages flottantes : $22 \%$

- Enclos : 13 \%

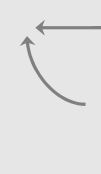

\section{$\mathbf{3} \mathbf{0 0 0}$ millions de larves}

produites en écloseries

Aliments pour poissons:

$>1000000$ t d'aliment artisanal (ic : $2,8: 1$ )

(60\% son de riz $+40 \%$ poisson frais)

+600000 t de granulés industriels (ic : 1,7:1)

(100 usines)

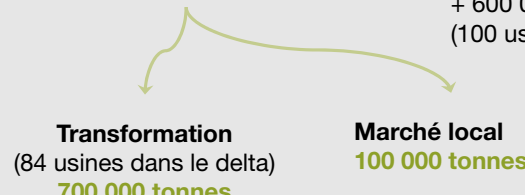

700000 tonnes

Filets congelés: 250000 tonnes

Exportation en Europe, Amérique, Asie
Sous-produits 450000 tonnes (carcasse)

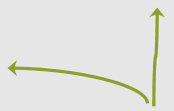

Transformations des sous-produits - Préparation de plats cuisinés - Extraction d'huile

- Production de farine de poisson

150000 personnes sont employées directement par la filière dont au moins 100000 aux élevages de grossissement + écloseries et 50000 au filetage/transformation des poissons.

Figure 7. Schéma de la filière Pangasius au Vietnam en 2006.

Figure 7. Representation of the Pangasius commodity chain in Vietnam in 2006.

tout à fait exceptionnel d'une filière de production, de transformation et d'exportation. Reposant principalement sur P. bypophthalmus, la production est ainsi passée en dix ans de 50000 à 800000 t en 2006 au Vietnam, qui a réalisé son objectif de production de $1000000 \mathrm{t}$ en 2007 (figure 8). S'agissant d'une espèce omnivore, à chaîne alimentaire courte, les coûts de production avec un aliment à faible teneur en protéines (22\% de protéines végétales pour l'aliment grossissement) demeurent faibles (0,6 US\$ $/ \mathrm{kg}$, soit $0,4 \mathrm{euro} / \mathrm{kg})$. Les filets congelés sont extrêmement compétitifs sur le marché international (6 à 8 euros/ $\mathrm{kg}$ au détail sur le marché français).

On peut raisonnablement prédire à ce poisson, ou aux autres du même type actuellement en cours de domestication dans le bassin du Mékong et en Indonésie, un avenir très prometteur sur le marché international. Cependant, une attention toute particulière devra être apportée à l'usage des antibiotiques afin d'éviter que ne s'aggravent les phénomènes d'antibiorésistance déjà constatés.

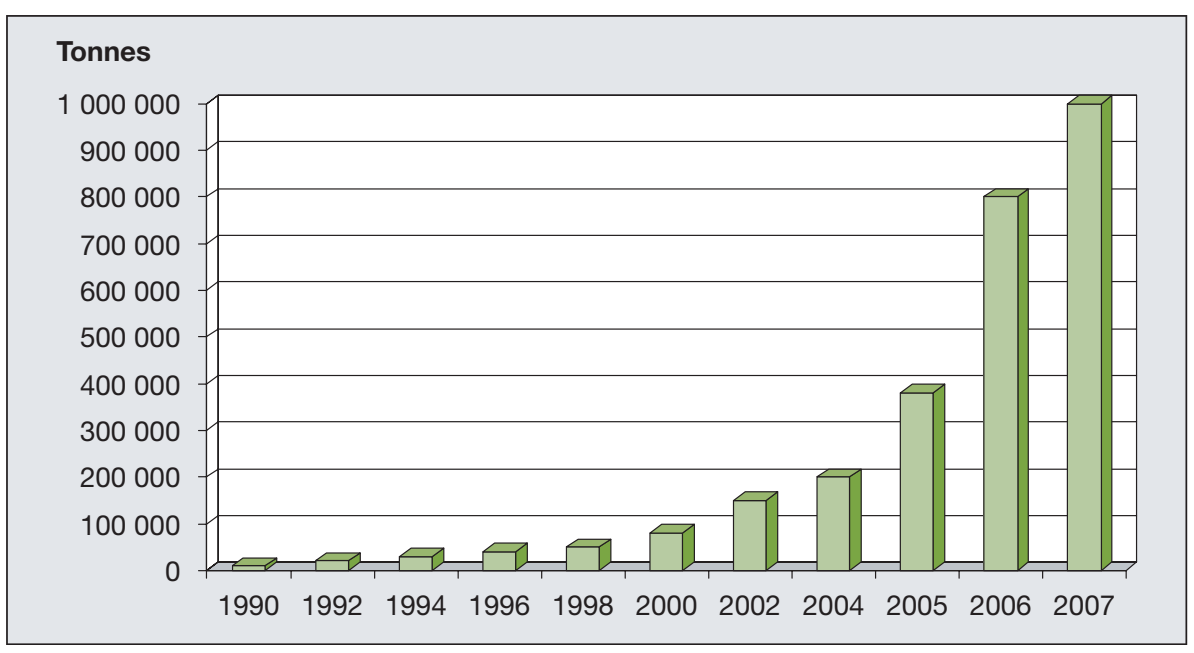

Figure 8. Évolution de la production de Pangasius sp. au Vietnam (en tonnes).

Figure 8. Variation in Pangasius sp. production in Vietnam (metric tons).

\section{Remerciements}

Les travaux exposés dans cet article se sont déroulés dans le cadre du programme Catfish Asia, coordonné par l'IRD et mené en partenariat avec le Research Institut for Freshwater Fisheries (RIFF) en Indonésie, et avec le Cirad et l'université de Can Tho au Vietnam (catfish Asia: characterisation, utilisation and maintenance of biological diversity for the diversification and sustainability of catfish culture in South-East Asia), soutenu par la Commission Européenne (DG XII, INCO-DC), 1996-2000). Au Vietnam, le Cirad et l'IRD ont, par ailleurs, développe une coopération avec une entreprise, AGIFISH, et l'UAF (Université agronomique et forestière de Thu Duc).

\section{Références}

Anonyme. Characterisation, utilisation and maintenance of biological diversity for the diversification and sustainability of catfish culture in south-east Asia. Final report to the European Commission, INCO-DC, contract No ERB IC18-CT96-0043, 2000.

Anonyme. Le poisson-chat du Mékong : quand le transfert talonne la recherche. In: le Cirad en 2000. Montpellier (France) : Cirad éditions, 2001.

Boonbrahm M. Report on the experiment on artificial fertilization of catfish Pangasius pangasius (Hamilton) at Nakorn Sawan fisheries station. Thai Fish Gaz 1959 ; 12 : 15-8.

Cacot P. Étude du cycle sexuel et maîtrise de la reproduction de Pangasius bocourti (Sauvage, 1880 ) et Pangasius hypophthalmus(Sauvage 1878) dans le delta du Mekong au Vietnam. Thèse de doctorat de I'Ina-PG, Ina-PG, CiradEMVT/Gamet, 1999.

Cacot $\mathrm{P}$, Legendre $\mathrm{M}$, Dan $\mathrm{TQ}$, et al. Induced ovulation of Pangasius bocourti (Sauvage, 1880 ) with a progressive hCG treatment. Aquaculture $2002 ; 213$ : 199-206.

Cacot P, Eeckhoutte P, Muon DT, et al. Spermiation hCG induced and management of milt in Pangasius bocourti. Aquaculture 2003 $215: 67-77$.

Cacot P, Lazard J. Domestication d'espèces de poissons-chats du Mékong de la famille des Pangasiidae. Inra Prod Anim 2004 ; 17 : 195-8.

Csavas I. Status and perspectives of culturing catfishes in East and South East Asia. FAO Aquaculture Newsletter 1994 ; 8 : 2-10.

Freud C, Richard J. Évaluation de l'impact de la maîtrise de la reproduction des poissons-chats dans le delta du Mékong sur le développement économique au Vietnam. Paris: CiradDirection Scientifique, 2002.

Guibert R. La pangasiculture dans le delta du Mékong (Vietnam): suivi de la qualité de l'eau en étangs et évaluation de la qualité des produits. Mémoire de fin d'études, Ina-PG, Cirad, Paris, 2003. 
Gustiano R. Taxonomy and phylogeny of Pangasiidae catfishes from Asia (Ostariophysi, Siluriformes). Ph.D., Thesis, Katholieke Universiteit, Leuven (Belgium), 2003.

Hardjamulia A, Djajadiredja R, Atmawinata S, Idris D. Pembenihan jambal siam (Pangasius sutchi) dengan suntikan ekstraks kelenjar hipofise ikan mas (Cyprinus carpio). Bull Pen Perik Darat 1981; 1 : 183-90.

Huillery AL. 2001. Analyse de la filière des poissons-chats (genre Pangasius) élevés dans le delta du Mékong (Vietnam). Mémoire de fin d'études pour l'obtention du grade d'ingénieur Ina-PG, Paris, 2001

Hung LT, Tam B, Cacot P, Lazard J. Larval rearing of the Mekong catfish, Pangasius bocourti (Pangasiidae, Siluroidei): substitution of Artemia nauplii with live and artificial feed. Aquat Living Resour 1999 ; 12 : 229-32.

Hung LT. Survey on technical and socioeconomical aspects of larval rearing of Pangasius hypophthalmus in Mekong delta. Communication présentée au "Final Meeting of the Catfish Asia Project", Bogor, Indonesia (15-20 May 2000). Bogor (Indonesia): CRIFI-IRD, 2000.

Hung LT, Nguyen AT, Lazard J. Effects of frequency and time of feeding on growth and feed utilization in two asian catfishes, Pangasius bocourti (Sauvage, 1880) and Pangasius hypophthalmus (Sauvage, 1878). J AquaTrop $2001 ; 16$ : 171-84.

Hung LT, Nguyen AT, Cacot P, Lazard J. Larval rearing of the Asian catfish, Pangasius bocourti, Pangasiidae: alternative feeds and weaning time. Aquaculture $2002 ; 212$ : 115-27.

Hung LT, Lazard J, Mariojouls C, Moreau Y. Comparison of starch utilization in fingerlings of two catfishes from the Mekong River (Pangasius bocourti Sauvage, 1880, Pangasius hypophthalmus Sauvage, 1878). Aquacult Nutr $2003 ; 9: 215-22$.

Hung LT, Suhenda N, Slembrouck J, Lazard J, Moreau Y. 2004. Comparison of dietary protein and energy utilization in three Asian catfishes (Pangasius bocourti, Pangasius hypophthalmus and Pangasius djambal). Aquacult Nutr $2004 ; 10: 317-26$.
Hung LT, Huy HPV, Truc LTT, Lazard J. Feeding practices and economic evaluation of Pangasiid catfish culture in Mekong delta, Vietnam. Communication présentée au $12^{\mathrm{e}}$ Symposium International de Nutrition et Alimentation des Poissons, Biarritz (28 may- $1^{\text {er }}$ juin 2006).

Huy LK, Duc HM, Hoa VP, Hao NV, Tuan N, Thu NKD. Artificial breeding of the catfish (Pangasius micronemus Bleeker). Ho-Chi-Minh City (Vietnam): Institute for Research in Aquaculture $\mathrm{n}^{\circ} 2,1990$.

ICCILMB (Interim Committee for Coordination of Investigations of the Lower Mekong Basin). Fisheries in the lower Mekong Basin (Review of the Fishery Sector in the Lower Mekong Basin). Bangkok (Thailand): Mekong Secretariat, 1992.

Kiem NV. Preliminary experiments for artificial propagation of catfish (Pangasius micronemus Block). Can-Tho, (Vietnam): Faculty of Fisheries (Can-Tho University); International Foundation for Science (Sweden), 1992.

Kristanto $\mathrm{AH}$, Slembrouck J, Legendre M. First sexual maturation and breeding cycle of Pangasius hypophthalmus (Siluriformes, Pangasiidae) reared in ponds. Indonesian Fish Res $J$ $2005 ; 11: 53-7$

Lazard J, Legendre M. Compte rendu de mis sion en Asie du Sud-Est (aquaculture continentale). Montpellier (France): Cirad; Orstom Gamet, 1993.

Lazard J, Cacot $P$. Systèmes de production aquacoles au Vietnam : situation, perspectives et enjeux de recherche. Cah Agric 1997; 6 : 445-54.

Legendre $M$, Subagja J, Slembrouck J. Absence of marked seasonal variations in sexual maturity of Pangasius hypophthalmus brooders held in ponds at the Sukamandi station (Java, Indonesia). In : Legendre M, Pariselle $\mathrm{A}$, eds. The biological diversity and aquaculture of clariid and pangasiid catfishes in south-east Asia. Proceedings of the mid-term workshop of the "Catfish Asia Project", 11-15 May 1998, Can Tho (Vietnam), 1999.

Legendre M, Pouyaud L, Slembrouck J, et al. Pangasius djambal: a new candidate species for fish culture in Indonesia. IARD J $2000 ; 22$ 1-14.
Legendre M, Slembrouck J, Subagja J, Kristanto $\mathrm{AH}$. Ovulation rate, latency period and ova viability after $\mathrm{GnRH}$ - or hCG-induced breeding in the Asian catfish Pangasius hypophthalmus (Siluriformes, Pangasiidae). Aquat Living Resour 2000 ; 13 : 145-51.

Lenormand S. Les Pangasiidae du delta du Mékong (Vietnam) : description préliminaire des pêcheries, éléments de biologie et perspectives pour une diversification des élevages. Mémoire de fin d'études pour l'obtention du grade d'ingénieur Ensar. Département environnement et exploitation des ressources naturelles, laboratoire halieutique, Ensar et IRD, 1996

MRC. Distribution and Ecology of Some Important Riverine Fish Species of the Mekong River Basin (MRC Technical Paper No. 10). Vientiane (Lao PDR) : Mekong River Commission, 2004.

Pouyaud L, Teugels GG, Gustiano R, Legendre M. Contribution to the phylogeny of pangasiid catfish (Siluriformes, Pangasiidae) based on allozymes and mitochondrial DNA. J Fish Biol $2000 ; 56: 1509-38$.

Sarter S, Nguyen HNK, Hung LT, Lazard J, Montet D. 2006. Antibiotic resistance in Gramnegative bacteria isolated from farmed catfish. Food Contr 2007 ; 18 : 1391-6.

Slembrouck J, Hung LT, Subagja J, Legendre M. Effects of prey quality, feeding level, prey accessibility and aeration on growth and survival of Pangasius hypophthalmus larvae (Siluroidei, Pangasiidae). In : Legendre $\mathrm{M}$, Pariselle A, eds. The biological diversity and aquaculture of clariid and pangasiid catfishes in south-east Asia. Proceedings of the mid-term workshop of the "Catfish Asia Project", 11-15 May 1998, Cantho (Vietnam), 1999.

Slembrouck J, Komarudin O, Maskur, Legendre M. Technical manual for the artificial propagation of the Indonesian Catfish, Pangasius djambal. Jakarta (Indonesia) : IRD-BKP Edition, 2003.

Subagja J, Slembrouck J, Hung LT, Legendre M. Larval rearing of an Asian catfish Pangasius hypophthalmus (Siluroidei, Pangasiidae): analysis of precocious mortality and proposition of appropriate treatments. Aquat Living Resour $1999 ; 12: 37-44$. 